\title{
UTILIZAÇÃO DE SENSORIAMENTO REMOTO EM ANÁLISES DE ALBEDO E TEMPERATURA DE SUPERFÍCIE EM LONDRINA - PR: CONTRIBUIÇÕES PARA ESTUDOS DE ILHA DE CALOR URBANA
}

\author{
NANCY LAURA RIOS GAMARRA ${ }^{1}$, MARCELO DE PAULA CORRÊA ${ }^{1}$, ADMIR CRÉSO DE \\ LIMA TARGINO ${ }^{2}$ \\ ${ }^{1}$ Universidade Federal de Itajubá (UNIFEI), Itajubá, MG, Brasil \\ ${ }^{2}$ Universidade Tecnológica Federal do Paraná (UTFPR), Londrina, PR, Brasil \\ nalariga@gmail.com,mpcorrea@unifei.edu.br, admirtargino@utfpr.edu.br
}

Recebido Junho de 2013 - Aceito Dezembro de 2013

\begin{abstract}
RESUMO
Este trabalho avaliou o albedo $\left(\alpha_{\mathrm{s}}\right)$ e a temperatura de superfície continental $\left(T_{\text {sup }}\right)$ provenientes de sensoriamento remoto, e a temperatura do ar $\left(T_{a r}\right)$ medida in situ no intuito de caracterizar a Ilha de Calor Urbana (ICU) na cidade de Londrina (Paraná). Foram utilizados dados de $\alpha_{\mathrm{s}}$ e $T_{\text {sup }}$ obtidos do sensor MODIS, e medições in situ de $T_{a r}$ realizadas entre junho e agosto de 2011 por sensores meteorológicos instalados em 13 locais na cidade. Os dados de $\alpha_{\mathrm{s}}$ e $T_{\text {sup }}$ foram utilizados para calcular um índice de urbanização (IU) na região e posteriormente comparado com $T_{a r}$. A análise dos dados de $\alpha_{\mathrm{s}}$ e $T_{\text {sup }}$ foi consistente com as medições in situ, evidenciando sua aplicabilidade na avaliação espaço-temporal de fenômenos acoplados continente-atmosfera, tais como a ICU. A mancha urbana da cidade de Londrina apresentou valores de IU compreendido entre 18 e 20, bem maiores que as áreas mais vegetadas (14 - 16). Nesse sentido, o IU demonstrou-se ser uma metodologia útil para representar a evolução espaço-temporal de áreas urbanas, o que pode propiciar uma avaliação detalhada do impacto da mudança de cobertura de solo no clima local e regional. Além disso, os resultados indicam que dados obtidos via satélite podem auxiliar grandemente na quantificação da ICU, quando dados in situ estiverem indisponíveis.

Palavras-chave: Albedo de superfície. Temperatura de superfície. Temperatura do ar. Índice de urbanização. Ilha de calor urbana.

\footnotetext{
ABSTRACT: USE OF REMOTE SENSING TO RETRIEVE SURFACE ALBEDO AND LAND SURFACE TEMPERATURE IN LONDRINA(PARANÁ): A CONTRIBUITION TO URBAN HEAT ISLAND STUDIES

This study retrieved surface albedo $\left(\alpha_{\mathrm{s}}\right)$ and land surface temperature $T_{\text {sur }}$ using remote sensing data in order to provide information for studies of Urban Heat Island (UHI) in the city of Londrina (Paraná). We have used $\alpha_{\mathrm{s}}$ and $T_{\text {sur }}$ data from the MODIS sensor, and in situ measurements of near-surface air temperature $\left(T_{\text {air }}\right)$ acquired between June and August 2011 at 13 sites across the city area. The albedo and surface temperature data were used to calculate an urbanization index (UI), and further compared with $T_{\text {air }}$. In general, the data analysis of $\alpha_{\mathrm{s}}$ and $T_{\text {sur }}$ was consistent with the in situ measurements, showing its applicability in the assessment of the spatial-temporal features of UHI. The urban area of Londrina showed UI values between 18 and 20, whilst vegetated areas had values between 14 and 16. The values are consistent with other studies, representing a useful methodology to assess the spatial-temporal evolution of urban areas. The index can also provide detailed information to assess the impact of land cover change on local and regional climate. Moreover, the results indicate that satellite data can greatly assist in the quantification of UHI when in situ air temperature data are unavailable. Keywords: Land surface albedo. Land surface temperature. Air temperature. Urban index. Urban heat island.
} 


\section{INTRODUÇÃO}

Segundo o Departamento das Nações Unidas para Assuntos Econômicos e Sociais (UN-PD, 2007), entre 1950 e 2010 a proporção da população brasileira vivendo em áreas urbanas passou de 36,2 \% para 86,5\%. Em geral, este crescimento populacional urbano foi acompanhado pela falta de planejamento associado ao rápido desenvolvimento da maioria das cidades.

Dentre os principais impactos causados pela urbanização, destacam-se a diminuição de áreas verdes, que são substituídas por materiais sintéticos (principalmente asfalto e concreto) utilizados nas construções e na pavimentação (Hu e Jia, 2010), a contaminação de corpos de água por sedimentos e metais pesados (Wildi et al., 2004), e a poluição do ar causados pela emissão de gases provenientes de atividades antropogênicas, tais como dióxido de enxofre, monóxido de carbono, ozônio, óxidos de nitrogênio, hidrocarbonatos, entre outros. (Asimakopoulos e Demosthenes, 2001; Barry e Chorley, 2010). Estes impactos geram estresse sobre ecossistemas como, por exemplo, alterações da diversidade de espécies animais e vegetais e modificações de paisagens devido à urbanização (Partecke et al., 2006; Mckinney, 2002). Além dessas alterações físicas associadas ao aumento da população urbana, somam-se os fatores relacionados com o consumo de recursos para satisfazer a demanda da população, como a produção de eletricidade, o uso de veículos automotores e produtos para refrigeração, que aumentam o calor antropogênico. O termo calor antropogênico refere-se a um tipo de energia gerada pela ação do ser humano, por meio de fontes móveis (combustão de combustíveis fosseis) e estacionárias (devido ao consumo de eletricidade e outros combustíveis em residências e em atividades industriais e comerciais), e pelo metabolismo humano e animal (Gartland, 2008; Ferreira et al., 2011; Taha, 1997).

As modificações das superfícies naturais para dar lugar à urbanização geram um fenômeno conhecido como Ilha de Calor Urbana (ICU), definida classicamente na literatura como uma anomalia térmica caracterizada pelo aumento da temperatura do ar $\left(T_{a r}\right)$ nas áreas centrais de uma cidade em relação à temperatura das áreas periféricas (Oke, 1973; Cermak et al., 1995, p. 81; Peng et al., 2012). A ICU é um dos impactos ambientais decorrentes da urbanização mais estudados na literatura, afetando, tanto as grandes metrópoles (Lombardo, 1985; Lucena et al., 2013; Yang et al., 2010; Dousset et al., 2011; Fujibe, 2011; Frey et al., 2011), quanto cidades de porte médio (aquelas com população entre 100 mil e 500 mil habitantes) (Targino et al., 2013; Amorim et al., 2009).

A principal causa da formação de ICU é a alteração do balanço de energia nas duas camadas (verticais) principais do ambiente urbano: camada do dossel urbano e camada limite urbana (Oke, 1973, 1982). A camada do dossel urbano (CDU) é definida como a coluna de ar que se estende desde o solo até a altura média dos edifícios (Gartland, 2008). Nesta camada se produzem as interações de microescala $\left(10^{-3}-10^{3} \mathrm{~m}\right)$ e as trocas de calor entre as paredes dos prédios, ruas, e ar adjacente. A segunda camada situa-se acima da CDU, e denomina-se camada limite urbana (CLU), com dimensão espacial de mesoescala $\left(10^{3}\right.$ $\left.-10^{5} \mathrm{~m}\right)$. Sua altura depende das características da rugosidade da superfície e da estabilidade atmosférica (Ferreira, 2010). Os diversos processos físicos provenientes das atividades urbanas (fluxos de calor e emissões de poluentes, por exemplo) que ocorrem próximo à superfície são misturados na CLU. Tanto a CDU, como a CLU são influenciadas pelas condições sinóticas de tempo (Barbirato et al., 2007).

Dentre as características de urbanização que contribuem ao desenvolvimento de ICU destacam-se a geometria urbana (Kruger et al., 2011), a substituição de áreas verdes por superfícies impermeáveis e com maior potencial de absorção de calor (Gallo et al., 1993; Weng et al., 2004), e incremento dos níveis de poluentes no ar (Lai e Cheng, 2009). A geometria urbana contribui com o aprisionamento da radiação incidente e dificulta a circulação do ar (Kruger et al., 2011). A falta de vegetação altera a partição dos fluxos de energia na superfície, reduzindo a evaporação do solo e o fluxo de calor latente e, consequentemente, aumentando o fluxo de calor sensível (Peng et al.,2012; Imhoff et al., 2010; Hu e Jia, 2010). Por outro lado, a poluição do ar colabora com o incremento do fluxo de radiação de onda longa entre $\mathrm{o}$ ar e as superfícies rugosas na CDU (absorção e reemissão), e com o aumento da absorção da radiação de onda curta na CLU em presença de aerossóis (Oke, 1982).

Além do uso do solo e de fatores antropogênicos, a ICU também é influenciada pela incidência de radiação solar e pelas condições sinóticas (Giridharan et al., 2007). Neste contexto, Targino et al. (2013) realizaram estudos da ICU na cidade de Londrina (Paraná) monitorando a $T_{a r}$ em 13 sítios selecionados de acordo com o uso de solo. O período de observação foi dominado por três condições sinóticas principais: frente fria (ocorrência de $21 \%$ do período observado), anticiclones frios (associados ou não a passagens frontal, $47 \%$ de ocorrência), e alta pressão estacionária ( $32 \%$ de ocorrência). A intensidade média da ICU é mínima $\left(1^{\circ} \mathrm{C}\right)$ durante períodos de frentes frias, e se intensifica progressivamente em situações de anticiclones frios $\left(3^{\circ} \mathrm{C}\right)$, e alta pressão estacionária $\left(6^{\circ} \mathrm{C}\right)$. Targino et al. (2013) enfatizaram que em condições de alta pressão estacionária, a incidência da radiação solar é maior e a velocidade do vento menor. Em tais condições a $T_{a r}$ é mais elevada em todos os sítios de monitoramento, e é influenciada principalmente pelas propriedades da superfície nos sítios com predominância de asfalto e telhado. Também foi observado que a intensidade da ICU é maior durante a noite e menor durante o dia, devido à 
baixa taxa de resfriamento de ambientes urbanos comparados com os sítios com predomínio de vegetação.

Entre as metodologias comumente utilizadas para identificar a ICU, destacam-se aquelas provenientes de medições in situ realizadas por termômetros fixos ou por termômetros montados em plataformas móveis para observar a distribuição espacial da $T_{a r}$. Assim, a este tipo de ICU denomina-se ICU atmosférica (Peng et al., 2012; Arnfield, 2003). Além das medições in situ, a utilização de observações remotas vem se destacando nas últimas quatro décadas, fornecendo uma alternativa viável para a quantificação da ICU por meio de sensores termais dispostos em diferentes plataformas, combinando satélites, aeronaves e estações em superfícies fixas (Voogt e Oke, 2003), através dos quais é possível obter temperaturas de superfícies $\left(T_{\text {sup }}\right)$, distinguindo-se deste modo a ICU superficial (ICUS) da ICU atmosférica.

A ICU atmosférica geralmente é medida dentro da CDU. Este tipo de ICU pode ser identificado mais facilmente durante a noite e em condições de céu claro e calmaria, quando a diferença de resfriamento radiativo entre as áreas urbanas e seus arredores menos urbanizados é máxima (Voogt e Oke, 2003; Targino et al, 2013). Por outro lado, os sensores remotos são mais adequados para avaliar a ICU diurna, uma vez que capturam a temperatura e outras características físicas (tais como albedo e emissividade) de um conjunto de superfícies heterogêneas dentro da área urbana, como, por exemplo, telhado, vegetação, pavimento, solo exposto e corpos de água, a partir da resposta espectral destas superfícies.

Uma das limitações da abordagem empregada nas metodologias de medição in situ diz respeito à resolução espacial das análises, que não conseguem identificar as heterogeneidades observadas nas intensidades de ICU em diferentes locais dentro da cidade, decorrentes dos diferentes tipos de uso do solo das áreas urbanas (Upmanis et al., 1998; Unger, 2004; Jin, 2012). Uma alternativa para amenizar essa deficiência seria obter medições in situ de $T_{a r}$ no perímetro urbano com uma resolução espacial alta (ou seja, com uma grande amostragem de medições no domínio de estudo), abrangendo diferentes categorias de uso de solo. No entanto, a aquisição e manutenção dos equipamentos de monitoramento torna essa abordagem muito custosa. Portanto, o sensoriamento remoto surge como uma técnica alternativa e de custo relativamente baixo na identificação da ICU, permitindo uma boa quantificação espaço-temporal de algumas características da superfície terrestre, tais como $T_{\text {sup }}$ e albedo $\left(\alpha_{\mathrm{s}}\right)$.

Diversos critérios diferenciam superfícies urbanas das superfícies não urbanizadas ou rurais. No caso de suas características físicas, o $\alpha_{\mathrm{s}}$ e a $T_{\text {sup }}$ são variáveis relevantes. Por exemplo, nas áreas urbanas se observam valores mais elevados de $T_{\text {sup }}$, do que em seus arredores não urbanizados.
Esta diferença está diretamente relacionada com a densidade das construções e com a mudança da cobertura vegetal (Hu e Jia, 2010). No entanto, a $T_{\text {sup }}$ por si só não é um indicador de urbanização, uma vez que $T_{\text {sup }}$ semelhantes entre as regiões central e periférica podem ser observadas em alguns lugares, como, por exemplo, na cidade de Phoenix, localizada em uma região desértica dos Estados Unidos (Jin et al., 2005). Por esta razão, Jin et al. (2005) desenvolveram um índice de urbanização (IU) no qual combinam dados de $T_{\text {sup }}$ e $\alpha_{\mathrm{s}}$ do sensor MODIS, permitindo assim distinguir as áreas urbanas das áreas rurais e, deste modo, facilitar a identificação delas em estudos de ICU e/ou planejamento urbano. Além do referido IU, As-syakur et al. (2012) descrevem outros índices para distinguir com boa acurácia superfícies urbanizadas das superfícies rurais, como por exemplo, o NDBI (Normalized Difference Built-up Index) (Zha et al., 2003), o IBI (Index-based Built-Up Index (Xu, 2008), o UI (Urban Index) (Kawamura et al., 1998) e o NDVI (Normalized Difference Vegetation Index) (Chen et al., 2006).

Com base na problemática supracitada, o presente trabalho propõe analisar dois produtos do sensor MODIS, que definem características de superfície $\left(\alpha_{\mathrm{s}}\right.$ e $\left.T_{\text {sup }}\right)$ sobre a cidade de Londrina (Paraná), com o objetivo de fornecer informações suplementares em estudos futuros da ICU na região. Além dos dados de $\alpha_{\mathrm{s}}$ e $T_{\text {sup }}$ provenientes de sensoriamento remoto, observações in situ de $T_{a r}$ medidas nas áreas urbana e suburbana da cidade serão utilizadas para uma avaliação de consistência dos dados obtidos por satélite. Adicionalmente, o índice de urbanização proposto por Jin et al. (2005) será aplicado sobre o domínio de estudo, no intuito de fornecer um mapeamento urbano para a distinção sistemática de áreas urbanas e rurais, o que facilitaria estudos futuros da ICU.

\section{DADOS E METODOLOGIA}

\subsection{Medições de temperatura do ar in situ}

Os dados de $T_{a r}$ utilizados neste estudo foram coletados durante o projeto Estudo da Ilha de Calor na Cidade de Londrina (ICALON) (Targino et al., 2013), no período de 07 de junho a 17 de agosto de 2011. Londrina é uma cidade de porte médio com cerca de 520 mil habitantes (IBGE, 2012), localizada no norte

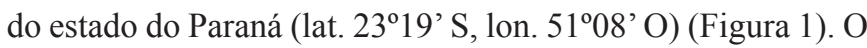
trabalho de campo do projeto ICALON foi dividido em duas etapas principais: (1) avaliação da cobertura do solo em áreas préselecionadas na mancha urbana da cidade e áreas circunvizinhas, e (2) medições de $T_{a r}$ em 13 locais selecionados na etapa anterior e classificados segundo a cobertura do solo (Figura 1).

Para a avaliação da cobertura do solo, Targino et al. (2013) extraíram imagens do Google Earth abrangendo uma área de $40.000 \mathrm{~m}^{2}$ ao redor de cada ponto a ser monitorado. As 


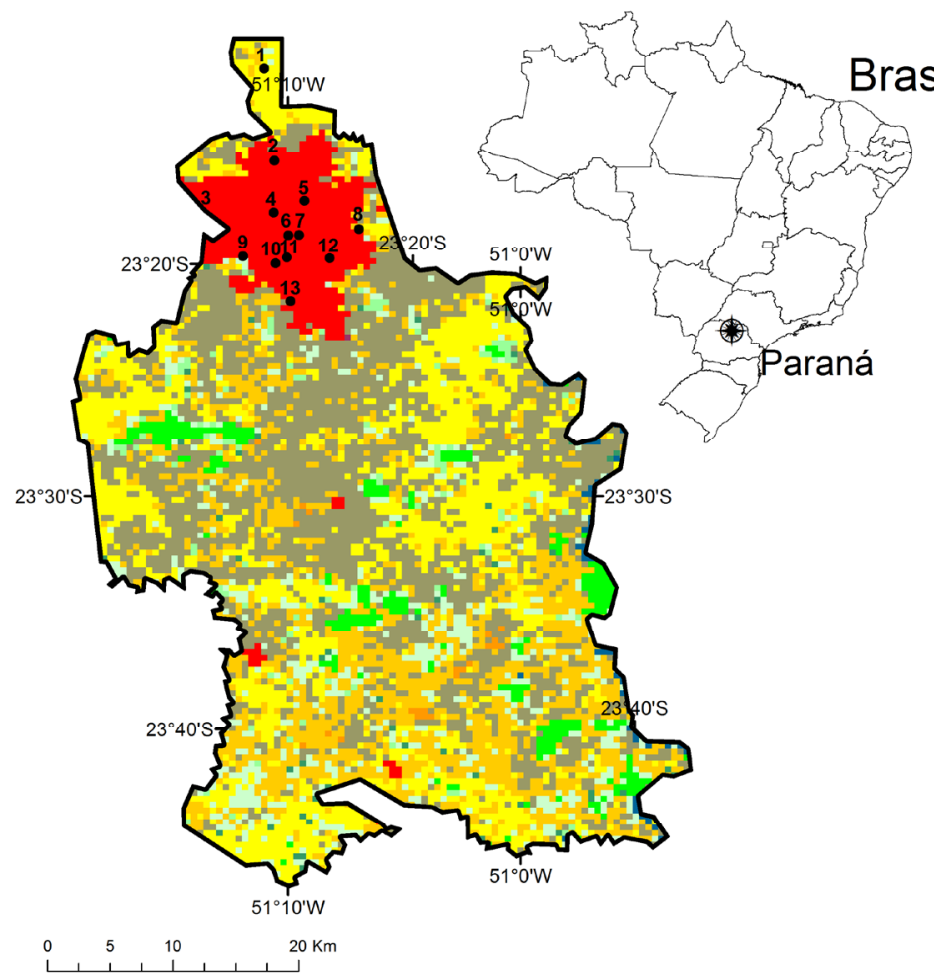

Uso de solo
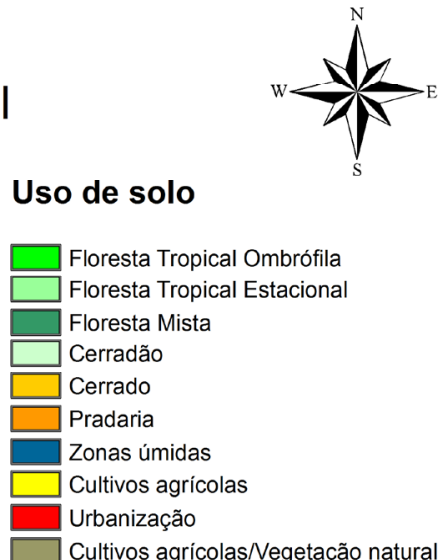

Pontos de monitoramento
1 - FMBRAPA Soja
2 - Rua Servino Freitas
3 - Parque de Exposição
4 - Rua Odilon Carvalho
5 - Av. Lucia Helena
6 - Rua Belo Horizonte
7 - Biblioteca Municipal de Londrina
8 - Univ. Tecnológica Federal do Paraná
9 - Univ. Estadual de Londrina
10 - Prédio Lessence
11 - IATE Clube
12 - Aeroporto de Londrina
13 - SIMEPAR

Figura 1 - Mapa de uso e ocupação do solo de Londrina e localização da área de estudo. Os pontos de monitoramento com seus respetivos nomes são indicados na porção norte do município. Para a classificação do uso de solo foi utilizado o produto anual MODIS MCD12Q1 de 500 m de resolução espacial, correspondente ao ano 2011. As cores e nomenclaturas seguem a classificação proposta pelo Programa Internacional da Geosfera - Biosfera (IGBP, 2013).

imagens foram analisadas com o software SPRING (Sistema de Processamento de Informações Georreferenciadas) (Câmara et al., 1996) por meio das seguintes etapas: (a) obtenção das imagens do Google Earth, (b) georreferenciamento com ajuda de uma imagem de alta resolução do Shuttle Radar Topography Mission da National Aeronautics and Space Administration (NASA), (c) classificação e quantificação (em \%) de cada uma das cinco categorias morfológicas em que foi classificada a cobertura do solo: vegetação, asfalto, telhado, água e solo exposto. Os resultados obtidos são apresentados na Tabela 1.

A temperatura do ar foi medida com sensores HOBOU23 (Onset Computer, Bourne, Massachusetts), com resolução temporal de $1 \mathrm{~min}$. O HOBO-U23 opera na faixa de temperatura entre -40 e $70{ }^{\circ} \mathrm{C}$, com resolução de $0,02{ }^{\circ} \mathrm{C}$ e precisão de 0,2 ${ }^{\circ} \mathrm{C}$. A EMBRAPA-Soja (EMB), o aeroporto de Londrina (LDB) e o Sistema Meteorológico do Paraná (SIMEPAR) forneceram dados de $T_{a r}$ medidos com instrumentos de suas estações meteorológicas. Os dados da EMB foram coletados com o sensor S-THB-M002 (Onset Computer, Bourne, Massachusetts), no sítio LDB com o sensor modelo 5190C (All Weather Inc., Sacramento, California) e no SIM com sensores Vaisala HMP110 (Vaisala, Helsinki), todos com $0,2{ }^{\circ} \mathrm{C}$ de precisão.

\subsection{Albedo de superfície a partir de sensoriamento remoto}

Os produtos do sensor MODIS (Moderate Resolution Imaging Spectroradiometer), acoplado aos satélites de órbita polar Terra e Aqua foram utilizados para obtenção dos dados de $\alpha_{s}$. Esses satélites são sincronizados com a órbita terrestre, de tal forma que a trajetória do satélite Terra é de norte a sul (passando sobre o equador pela manhã), enquanto a trajetória do Aqua é de sul para o norte (passando sobre o equador à tarde). Ambos os sensores cobrem toda a superfície terrestre de um a dois dias, e a aquisição de dados pode ser feita em 36 bandas espectrais (Anderson et al., 2003).

Para realizar o cálculo de $\alpha_{\mathrm{s}}$, foram extraídos dados de um conjunto de imagens identificadas com o código MCD43B3, de $1000 \mathrm{~m}$ de resolução espacial e 16 dias de resolução temporal. Cada imagem é um mosaico de 2400 x 2400 pixels, chamado grânulo. Os grânulos estão disponibilizados no site da NASA, como parte do Sistema de Dados e Informações do Sistema de Observações Terrestres (EOSDIS, 2009). O MODIS produz 288 grânulos a cada 24 horas, cobrindo aproximadamente 2.340 $\mathrm{km}$ por $2.030 \mathrm{~km}$ (Anderson et al., 2003). Cada grânulo foi 
Tabela 1 - Sítios de monitoramento e suas respectivas classificações de uso do solo (\%) em uma área de $40.000 \mathrm{~m}^{2}$ ao redor de cada ponto. As siglas dos sítios avaliados são apresentadas entre parênteses.

\begin{tabular}{lccccc}
\hline \multirow{2}{*}{ Sítios } & \multicolumn{5}{c}{ Uso do solo } \\
\cline { 2 - 6 } & Vegetação & Telhado & Asfalto & Água & Solo Exposto \\
\hline Prédio Lessence (LES) & 7 & 47 & 43 & 1 & 2 \\
Rua Odilon Carvalho (ODI) & 16 & 46 & 38 & 0 & 0 \\
Av. Lucia Helena (LUC) & 17 & 60 & 22 & 0 & 0 \\
Aeroporto (LDB) & 19 & 17 & 64 & 0 & 0 \\
Rua Belo Horizonte (BHZ) & 21 & 61 & 17 & 1 & 0 \\
Pq. de Exposições (PQE) & 31 & 22 & 28 & 0 & 18 \\
Rua Servino Freitas (SER) & 31 & 49 & 12 & 0 & 8 \\
IATE Clube (IAT) & 33 & 14 & 20 & 32 & 0 \\
Biblioteca Municipal (BIB) & 38 & 40 & 21 & 0 & 0 \\
Universidade Tecnológica & 51 & 9 & 24 & 0 & 16 \\
Federal do Paraná (UTF) & & & & & 0 \\
Universidade Estadual de & 69 & 17 & 13 & 0 & 0 \\
Londrina (UEL) & 83 & 6 & 11 & 0 & 10 \\
EMBRAPA Soja (EMB) & 86 & 2 & 0 & 2 & \\
SIMEPAR (SIM) & & & & & 0 \\
\hline Fonte: Targino et al.(2013) & & & & \\
\end{tabular}

Fonte: Targino et al.(2013).

escolhido de forma a incluir as coordenadas de Londrina (Figura 1). Mediante o software MODIS Reprojection Tool (MRT), disponível no pacote computacional do produto MCD43B3 (Schaaf, 2006), o grânulo é convertido do formato HDF para TIF, de modo a facilitar o processamento das imagens com outros pacotes computacionais. O seguinte passo foi mudar da projeção sinusoidal para projeção geográfica (latitude/ longitude).

Em seguida, calculou-se $\alpha_{\mathrm{s}}$ através da combinação linear de três quantidades: White Sky Albedo (WSA) ou em tradução literal "albedo de céu claro", Black Sky Albedo (BSA) ou "albedo de céu escuro" e a fração difusa (FD), de acordo com a Equação 1:

$$
\alpha_{s}=(W S A \times F D)+(B S A \times(1-F D))
$$

em que WSA é a componente de iluminação difusa isotrópica na ausência da componente de iluminação direta, e o BSA é a componente de iluminação direta na ausência da componente de iluminação difusa (dependente apenas do ângulo zenital solar). WSA e BSA são avaliadas no intervalo de 0,25 a $4,00 \mu \mathrm{m}$.

$\mathrm{Na}$ obtenção do $\alpha_{\mathrm{s}}$ a partir da interpolação do WSA e BSA, deve-se considerar a fração da radiação solar que é difundida pelos constituintes atmosféricos (FD) (Lewis e Barnsley, 1994). Portanto, FD na Equação 1 depende da profundidade óptica do aerossol (Strahler et al., 1999) e é obtida da tabela SKYL LUT (do inglês Sky Light Look-Up Table), disponível no pacote computacional do produto MCD43B3. A SKYL LUT é uma tabela de consulta que contém os valores da
FD, em função dos tipos de aerossóis (continentais e marítimos), profundidade óptica ( 0 a 1 , com variação de 0,02$)$ e ângulo zenital solar $\left(0^{\circ}\right.$ a $89^{\circ}$, com resolução de $\left.1^{\circ}\right)$.

Neste trabalho assume-se o tipo de aerossol continental, e a profundidade óptica do aerossol foi obtida na página web GIOVANNI (acrônimo do inglês, GES - DISC (Goddard Earth Science Data and Information Center) Interactive Online Visualization ANd aNalysis Infraestructure) (Acker e Leptoukh, 2007). GIOVANNI é uma página web desenvolvida pelo Centro Goddard de Informações e Serviços de Dados referentes às Ciências da Terra (GES - DISC), vinculado à NASA, fornecendo dados de sensoriamento remoto a nível global, de maneira que os usuários possam visualizar, analisar e acessar os dados online, sem necessidade de descarregálos. Neste estudo em particular, a página web GIOVANNI foi utilizada para obter os valores da profundidade óptica do aerossol na banda espectral de $550 \mathrm{~nm}$, fornecidos pelos produtos MOD08_M3.051 e MYD08_M3.051, do MODIS/ Terra e MODIS/Aqua, respectivamente. Finalmente, calculou-se o ângulo zenital solar a partir da Equação 2 (Ometto, 1981):

$$
\cos (\theta)=[\operatorname{sen}(\text { lat }) \times \operatorname{sen}(d)]+[\cos (\text { lat }) \times \cos (d) \times \cos (h)]
$$

em que $\theta$ é ângulo zenital solar; lat é a latitude do sítio selecionado; $d$ é a declinação solar calculada por $23,45 \operatorname{sen}[(360 / 365) \times(D D A-80)] ; D D A$ refere-se ao dia do ano (1-365); e $h$, o ângulo horário, calculado por (horalocal - 12)x15. 


\subsection{Temperatura de superfície a partir de sensoriamento remoto}

Para as análises da $T_{\text {sup }}$, utilizaram-se os produtos MYD11A1 e MOD11A2 dos sensores MODIS/Aqua e MODIS/Terra, respectivamente. MYD11A1 e MOD11A2 estão disponibilizados em grânulos de 1200 x 1200 pixels, com resolução horizontal de 1000 m (EOSDIS, 2009). Ambos os produtos são gerados em condições de céu claro utilizando o algoritmo generalized split -window, método amplamente utilizado para obter $T_{\text {sup }}$ a partir de imagens de satélites.

Para se obter $T_{\text {sup }}$ com o método split - Window, se requer informações de pelo menos duas bandas espectrais. No caso do MODIS, as bandas $31(10,780-11,280 \mu \mathrm{m})$ e 32 $(11,770-12,270 \mu \mathrm{m})$ são utilizadas nesse método. Isto porque a emissividade espectral característica das coberturas de solos são relativamente estáveis para o intervalo de comprimento de onda compreendida entre 10,5 e 12,5 $\mu \mathrm{m}$ (Wan e Dozier, 1996). Além das bandas, outros fatores são inseridos no algoritmo para se obter a temperatura de superfície, tais como as emissividades médias das bandas a serem tratadas, diferenças entre essas emissividades e as condições da atmosfera e superfície que por sua vez dependem do ângulo de visada, conteúdo de vapor de água na coluna atmosférica, e temperatura do ar próximo à superfície. Os dados de emissividades para a aplicação do algoritmo generalized split-window na obtenção dos produtos MODIS $T_{\text {sup }}$, estão baseados nas emissividades modeladas para 14 tipos de coberturas de solos fornecido pelo produto MODIS land-cover. Os coeficientes relativos à ampla gama de superfícies e condições atmosféricas são obtidos mediante técnicas de regressão linear a partir de dados simulados para os produtos do MODIS e disponibilizados nas tabelas LUT (Coll et al., 2005; Wan et al., 2004).

A $T_{\text {sup }}$ extraída do produto MYD11A1 com resolução temporal diária foi utilizada para relacioná-la com a série temporal de $T_{a r}$ medida em cada um dos 13 sítios avaliados neste estudo. Por outro lado, para a avaliação espacial de $T_{\text {sup }}$ e para o cálculo do índice de urbanização que será detalhado na próxima seção, utilizou-se o produto MOD11A2, que possui resolução temporal de 8 dias. Uma vez que os dados de $\alpha_{\mathrm{s}}$ disponíveis no produto MCD43B3 possuem resolução temporal de 16 dias, a escolha do produto MOD11A2 para a avaliação espacial de $T_{s u p}$ deve-se à consistência e facilitação dos cálculos exigidos no índice de urbanização, que relacionam $\alpha_{\mathrm{s}}$ e $T_{\text {sup }}$.

A $T_{\text {sup }}\left({ }^{\circ} \mathrm{C}\right)$ correspondente a cada grânulo é obtido pela equação:

$$
T_{\text {sup }}=\left(b_{1} \times 0,02\right)-273,15
$$

em que $b_{1}$ é o grânulo original MYD11A1 ou MOD11A2 e 0,02 é o fator de escala desses produtos.

\section{4 Índice de urbanização}

Existe na comunidade científica uma ampla variedade de imagens de distintos satélites e sensores com diferentes resoluções espaciais, espectrais e temporais que são utilizadas para mapear o uso e cobertura de solo em nível local, regional e global (Dousset e Goumerlon, 2003; Nichol, 1998; Cihlar, 2000; McRoberts et al., 2002; Friedl et al., 2010). As combinações apropriadas das bandas espectrais das imagens de satélites constituem uma valiosa ferramenta para identificar determinadas superfícies e suas mudanças. Porém, o processamento das imagens para a identificação de superfícies urbanas em grande escala torna-se um trabalho extremamente árduo e demorado, dado que necessita o reconhecimento visual e classificação "manual" por um usuário das superfícies envolvidas.

Visando uma solução prática para este problema, Jin et al. (2005) propôs uma combinação linear das variáveis $\alpha_{\mathrm{s}} \mathrm{e} T_{\text {sup }}$, que possibilita diferenciar de forma sistemática as superfícies urbanas das superfícies rurais. Por exemplo, o $\alpha_{\mathrm{s}}$ das superfícies urbanas tende a ser geralmente menor do que o das áreas rurais, o que implica em uma maior absorção de energia quando estas superfícies são expostas à radiação solar e, consequentemente, provoca um aumento de $T_{\text {sup }}$ e da temperatura do ar adjacente.

$\mathrm{O}$ índice de urbanização $(I U)$ proposto por Jin et al. (2005) é calculado como:

$$
I U_{i}=\left(1-\alpha_{s i}\right) \times T_{\sup i}
$$

em que o subscrito $i$, representa o i-ésimo pixel das imagens que contém $\alpha_{\mathrm{s}}$ e $T_{\text {sup. }}$. Os valores de IU são mais elevados em áreas urbanas onde $T_{\text {sup }}$ é maior e $\alpha_{\mathrm{s}}$ é menor, comparado com áreas rurais.

\section{RESULTADOS E DISCUSSÃO}

Para facilitar as análises desta seção, os resultados e figuras foram agrupados segundo dois critérios: i) de acordo com as porcentagens de vegetação contidas nas áreas dos sítios onde foram instalados os sensores para mediação de $T_{a r}$, e ii) com relação aos horários das passagens dos satélites (diurno e noturno). Adicionalmente foram selecionados dois sítios mais representativos de diferentes usos de solo: o sítio LES, que é predominantemente urbano e o sítio SIM com características suburbanas e com elevada influência de áreas vegetais (Figura 2, Tabela 1).

A Figura 3 apresenta as relações entre $T_{a r}$ e $T_{\text {sup }}$, juntamente com os coeficientes de correlação e as equações da reta de regressão linear. Cada um dos pontos apresentados nos diagramas de dispersão representa os dados de $T_{a r}$ e $T_{\text {sup }}$, abrangendo o período de 07 de junho até 17 de agosto de 2011 (período de medições da $T_{a r}$ do projeto ICALON). Nesta 

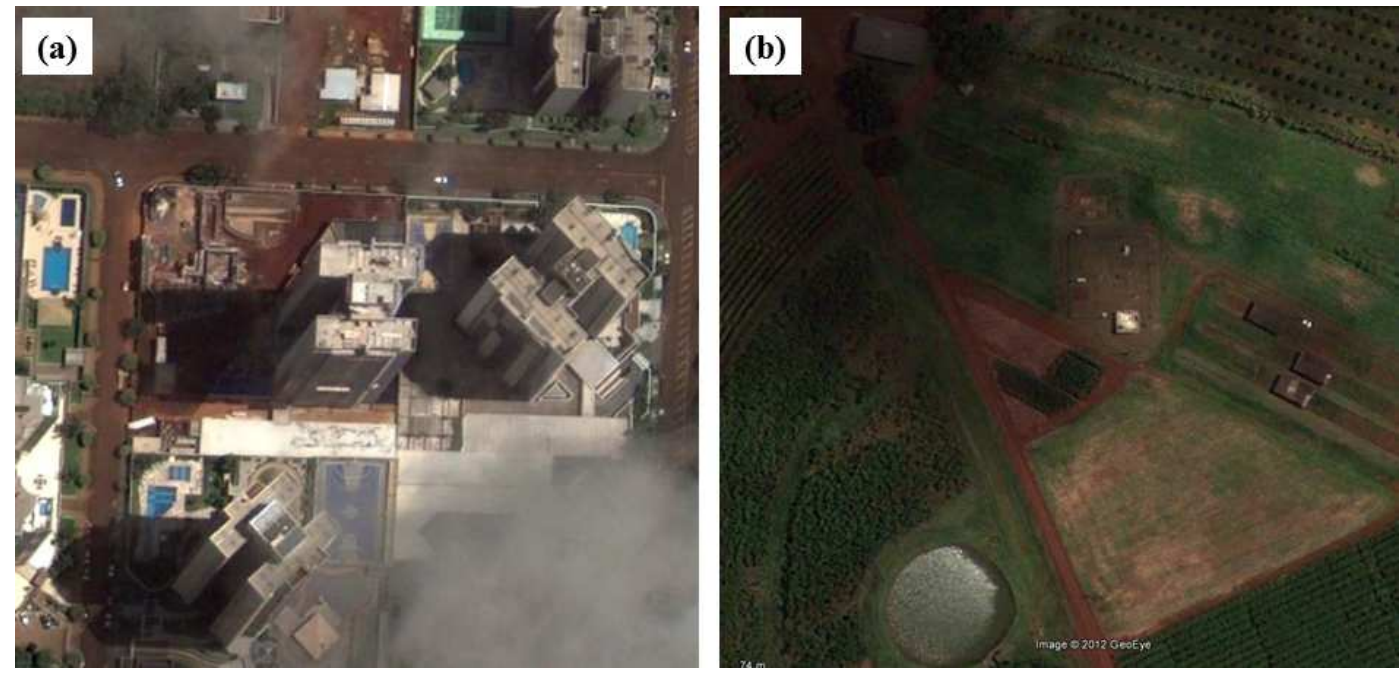

Figura 2 - Vista aérea dos sítios a) LES e b) SIM obtidas das imagens Google Earth.

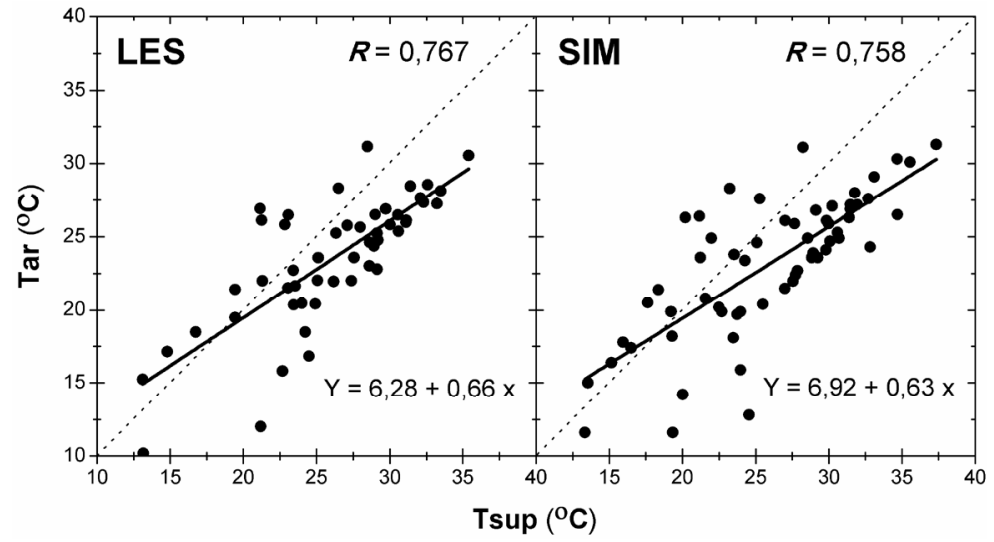

Figura 3 - Diagrama de dispersão entre a temperatura do ar próximo à superfície e temperatura da superfície no período diurno, para um sítio localizado na mancha urbana de Londrina (LES) e um sítio rural (SIM). A correlação de Pearson é indicada no canto superior direito, e a equação da reta de regressão (linha contínua em negrito) é indicada no canto inferior direito. A linha diagonal pontilhada indica uma correlação igual a 1.

análise, utilizou-se a $T_{\text {sup }}$ extraída do satélite Aqua (vide seção 2.3). Como as passagens diárias do satélite Aqua próximo a Londrina ocorrem no período diurno entre 13 e 15 horas local, e no período noturno entre 01 e 03 horas local, os dados in situ de $T_{a r}$ usados nessa comparação foram escolhidos como aqueles com os horários mais próximos das passagens do satélite. O sítio identificado com a sigla LES é um sítio urbano no qual $7 \%$ de sua área está coberta com vegetação e $90 \%$ com superfícies impermeáveis (asfalto e telhado, Tabela 1). Por outro lado, o sítio SIM, localizado na fronteira sul da mancha urbana, apresenta uma área com $86 \%$ de cobertura vegetal, com predomínio de culturas agrícolas. Em ambos os sítios se observa uma forte correlação entre as variáveis $(\mathrm{R}>0,75)$ (Figura 3). É possível identificar também que a $T_{\text {sup }}$ supera a $T_{a r}$ em $75 \%$ das medições. Isto é esperado, uma vez que durante o dia as superfícies absorvem mais radiação solar, fazendo com que a $T_{\text {sup }}$ seja maior que a $T_{a r}$.
Ao contrário do que constatou-se no período diurno, as observações no período noturno mostram que $T_{a r}$ é maior que $T_{\text {sup }}$ (Figura 4). Isto se explica pela perda de energia da superfície através do fluxo de radiação de onda longa, e absorção de parte dessa radiação pelo ar adjacente. As duas variáveis apresentam uma forte correlação $(R>0,70)$ no período noturno para ambos os sítios (Figura 4).

Os valores de $T_{\text {sup }}$ e $T_{a r}$ para os sítios LES e SIM estão ilustrados nas Tabela 2 (período diurno) e Tabela 3 (período noturno). Dado que as medições de $T_{a r}$ começaram na segunda quinzena do mês de junho até a primeira quinzena de agosto, optou-se por apresentar ilustrativamente somente o mês de julho, o qual encontra-se completo. Durante o dia, $T_{s u p}$ supera $T_{a r}$ em $75 \%$ do período de observação em contraste com o período noturno, no qual $T_{a r}$ supera $T_{\text {sup }}$ em todo o período de observação. Essa diferença de $T_{\text {sup }}$ e $T_{a r}$ durante o dia e a noite se observa em todo o período de monitoramento e para os 13 sítios selecionados. 


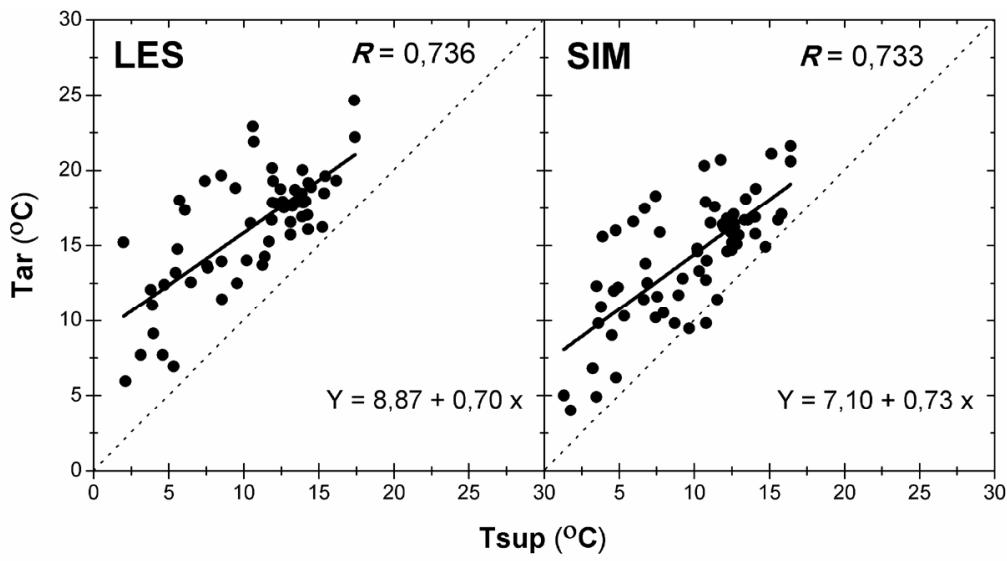

Figura 4 - Mesmo que Figura 3, exceto para o período noturno.

Tabela 2 - Temperatura de superfície (Tsup), e Temperatura do ar (Tar) e diferenças (Tsup-Tar ) no período diurno para um sítio localizado na mancha urbana de Londrina (LES) e um sítio rural (SIM).

\begin{tabular}{|c|c|c|c|c|c|c|}
\hline \multirow{2}{*}{$\begin{array}{c}\text { Julho/2011 } \\
\text { Dia }\end{array}$} & \multicolumn{3}{|c|}{ LES } & \multicolumn{3}{|c|}{ SIM } \\
\hline & $T_{\text {sup }}\left({ }^{\circ} \mathrm{C}\right)$ & $T_{a r}\left({ }^{\circ} \mathrm{C}\right)$ & Dif. & $T_{\text {sup }}\left({ }^{\circ} \mathrm{C}\right)$ & $T_{a r}\left({ }^{\circ} \mathrm{C}\right)$ & Dif. \\
\hline 01 & - & 18,485 & - & - & 18,4 & - \\
\hline 02 & 16,75 & 18,485 & $-1,735$ & 16,49 & 17,4 & $-0,91$ \\
\hline 03 & - & 17,153 & - & - & 17 & - \\
\hline 04 & 14,81 & 17,153 & $-2,343$ & 15,15 & 16,4 & $-1,25$ \\
\hline 05 & - & 17,558 & - & - & 17,7 & - \\
\hline 06 & - & 20,031 & - & - & 19,8 & - \\
\hline 07 & 21,31 & 22,011 & $-0,701$ & 21,57 & 20,8 & 0,77 \\
\hline 08 & 23,55 & 21,652 & 1,898 & 17,63 & 20,5 & $-2,87$ \\
\hline 09 & 26,17 & 21,963 & 4,207 & 25,49 & 20,4 & 5,09 \\
\hline 10 & 31,13 & 26,134 & 4,996 & 30,71 & 24,9 & 5,81 \\
\hline 11 & 29,73 & 26,891 & 2,839 & 27,01 & 26,1 & 0,91 \\
\hline 12 & 21,15 & 26,916 & $-5,766$ & 20,19 & 26,3 & $-6,11$ \\
\hline 13 & 32,09 & 27,653 & 4,437 & 31,47 & 27,2 & 4,27 \\
\hline 14 & 30,57 & 26,475 & 4,095 & 29,11 & 26,8 & 2,31 \\
\hline 15 & 33,23 & 27,259 & 5,971 & 31,93 & 27,2 & 4,73 \\
\hline 16 & 31,41 & 28,468 & 2,942 & 30,23 & 27,1 & 3,13 \\
\hline 17 & 33,49 & 28,122 & 5,368 & 32,69 & 27,6 & 5,09 \\
\hline 18 & 19,43 & 19,484 & $-0,054$ & 19,29 & 18,2 & 1,09 \\
\hline 19 & 25,11 & 23,569 & 1,541 & 24,27 & 23,4 & 0,87 \\
\hline 20 & 26,51 & 28,32 & $-1,81$ & 25,27 & 27,6 & $-2,33$ \\
\hline 21 & - & 18,271 & - & - & 17,7 & - \\
\hline 22 & 23,41 & 22,729 & 0,681 & 18,35 & 21,4 & $-3,05$ \\
\hline 23 & 19,43 & 21,413 & $-1,983$ & 19,21 & 19,9 & $-0,69$ \\
\hline 24 & 28,61 & 23,016 & 5,594 & 27,87 & 22,7 & 5,17 \\
\hline 25 & 23,07 & 26,475 & $-3,405$ & 21,23 & 23,6 & $-2,37$ \\
\hline 26 & 30,79 & - & - & 30,09 & 24,7 & 5,39 \\
\hline 27 & 27,11 & 25,768 & 1,342 & 27,67 & 25,9 & 1,77 \\
\hline 28 & 30,03 & 25,841 & 4,189 & 29,85 & 26,1 & 3,75 \\
\hline 29 & 32,59 & 28,568 & 4,022 & 31,77 & 28 & 3,77 \\
\hline 30 & - & 19,365 & - & - & 19,4 & - \\
\hline 31 & - & 19,222 & - & - & 18,6 & - \\
\hline
\end{tabular}

A Tabela 4 ilustra os valores dos coeficientes de correlação linear $(\mathrm{R})$ e os coeficientes de determinação $\left(\mathrm{R}^{2}\right)$ entre $T_{a r}$ e $T_{\text {sup }}$ para os 13 sítios monitorados durante o projeto ICALON. Na maioria dos sítios se observa uma forte correlação $(R>0,70)$ e um alto valor de coeficiente de determinação $(0,45$
$\left.<\mathrm{R}^{2}<0,70\right)$ entre $T_{a r}$ e $T_{\text {sup }}$. No período diurno, os sítios BHZ e EMB apresentam valores de $\mathrm{R}>0,80$, com $\mathrm{R}^{2}$ entre 0,65 e 0,67 , sendo estes sítios bem representativos da superfície urbana e rural, respectivamente. O sítio BHZ possui maior quantidade de asfalto e telhado (78\%, Tabela 1$)$ do que vegetação $(21 \%)$, 
Tabela 3 - Mesmo que Tabela 2, exceto para o período noturno.

\begin{tabular}{|c|c|c|c|c|c|c|}
\hline \multirow{2}{*}{$\begin{array}{c}\text { Julho/2011 } \\
\text { Dia }\end{array}$} & \multicolumn{3}{|c|}{ LES } & \multicolumn{3}{|c|}{ SIM } \\
\hline & $T_{\text {sup }}\left({ }^{\circ} \mathrm{C}\right)$ & $T_{a r}\left({ }^{\circ} \mathrm{C}\right)$ & Dif. & $T_{\text {sup }}\left({ }^{\circ} \mathrm{C}\right)$ & $T_{a r}\left({ }^{\circ} \mathrm{C}\right)$ & Dif. \\
\hline 01 & 13,11 & 16,558 & $-3,448$ & 12,95 & 15,7 & $-2,75$ \\
\hline 02 & - & 16,249 & - & 4,77 & 16 & $-11,23$ \\
\hline 03 & 6,07 & 17,368 & $-11,298$ & 5,95 & 16,6 & $-10,65$ \\
\hline 04 & 3,91 & 11,029 & $-7,119$ & 3,79 & 10,9 & $-7,11$ \\
\hline 05 & 3,81 & 12,074 & $-8,264$ & 3,61 & 9,8 & $-6,19$ \\
\hline 06 & 7,61 & 13,522 & $-5,912$ & 7,53 & 11,6 & $-4,07$ \\
\hline 07 & - & 16,368 & - & - & 14,5 & - \\
\hline 08 & 7,57 & 13,666 & $-6,096$ & 6,63 & 11,4 & $-4,77$ \\
\hline 09 & 6,47 & 12,558 & $-6,088$ & 5,33 & 10,3 & $-4,97$ \\
\hline 10 & 1,99 & 15,223 & $-13,233$ & 3,49 & 12,3 & $-8,81$ \\
\hline 11 & 12,11 & 17,819 & $-5,709$ & 11,99 & 16,2 & $-4,21$ \\
\hline 12 & 11,97 & 19,318 & $-7,348$ & 11,09 & 16,5 & $-5,41$ \\
\hline 13 & 7,41 & 19,318 & $-11,908$ & 7,43 & 18,3 & $-10,87$ \\
\hline 14 & 13,87 & 18,485 & $-4,615$ & 12,43 & 16,9 & $-4,47$ \\
\hline 15 & 11,87 & 16,701 & $-4,831$ & 10,19 & 14,8 & $-4,61$ \\
\hline 16 & 11,93 & 17,867 & $-5,937$ & 10,21 & 14,6 & $-4,39$ \\
\hline 17 & 15,43 & 19,627 & $-4,197$ & 13,43 & 18,1 & $-4,67$ \\
\hline 18 & 11,91 & 20,174 & $-8,264$ & 11,77 & 20,7 & $-8,93$ \\
\hline 19 & 14,29 & 16,082 & $-1,792$ & 14,07 & 15,8 & $-1,73$ \\
\hline 20 & 14,43 & 18,961 & $-4,531$ & 14,05 & 16,9 & $-2,85$ \\
\hline 21 & 17,37 & 24,629 & $-7,259$ & 16,41 & 21,6 & $-5,19$ \\
\hline 22 & 12,59 & 17,938 & $-5,348$ & 12,61 & 17,1 & $-4,49$ \\
\hline 23 & 15,23 & 16,225 & $-0,995$ & 14,75 & 14,9 & $-0,15$ \\
\hline 24 & 13,11 & 15,724 & $-2,614$ & 12,49 & 14,7 & $-2,21$ \\
\hline 25 & 13,89 & 16,915 & $-3,025$ & 13,03 & - & - \\
\hline 26 & 14,49 & 18,889 & $-4,399$ & 13,57 & 16,7 & $-3,13$ \\
\hline 27 & 13,41 & 18,723 & $-5,313$ & 12,65 & 16,3 & $-3,65$ \\
\hline 28 & 13,91 & 20,031 & $-6,121$ & 12,43 & 15,9 & $-3,47$ \\
\hline 29 & 13,59 & 17,915 & $-4,325$ & 12,37 & 16,5 & $-4,13$ \\
\hline 30 & 10,61 & 22,896 & $-12,286$ & 10,75 & 17,9 & $-7,15$ \\
\hline 31 & 12,67 & 17,534 & $-4,864$ & 12,55 & 16,6 & $-4,05$ \\
\hline
\end{tabular}

Tabela 4 - Coeficientes de correlação linear (R) e coeficiente de determinação $\left(\mathrm{R}^{2}\right)$ entre e para cada um dos sítios avaliados nesse estudo, em horários diurno e noturno.

\begin{tabular}{lcccc}
\hline \multirow{2}{*}{ Sítios } & \multicolumn{2}{c}{ Diurno } & \multicolumn{2}{c}{ Noturno } \\
& $\mathbf{R}$ & $\mathbf{R}^{\mathbf{2}}$ & $\mathbf{R}$ & $\mathbf{R}^{\mathbf{2}}$ \\
\hline LES & 0,767 & 0,580 & 0,736 & 0,534 \\
ODI & 0,723 & 0,512 & 0,740 & 0,538 \\
LUC & 0,788 & 0,612 & 0,788 & 0,613 \\
LDB & 0,722 & 0,513 & 0,700 & 0,482 \\
BHZ & 0,811 & 0,651 & 0,690 & 0,468 \\
PQE & 0,736 & 0,533 & 0,803 & 0,640 \\
SER & 0,680 & 0,452 & 0,755 & 0,562 \\
IAT & 0,769 & 0,584 & 0,676 & 0,448 \\
BIB & 0,772 & 0,588 & 0,684 & 0,457 \\
UTF & 0,733 & 0,528 & 0,724 & 0,515 \\
UEL & 0,794 & 0,622 & 0,820 & 0,665 \\
EMB & 0,824 & 0,672 & 0,702 & 0,484 \\
SIM & 0,758 & 0,567 & 0,733 & 0,530 \\
\hline
\end{tabular}


enquanto que no sítio EMB essas quantidades se invertem, com menores porcentagens de asfalto e telhado (17\%) e abundância de vegetação (83\%). No período noturno, os sítios PQE e UEL (característicos de superfície urbana e rural, respectivamente) também apresentam forte correlação entre $T_{a r}$ e $T_{\text {sup }}$.

Em resumo, observou-se que todos os sítios analisados apresentaram forte correlação linear entre suas respectivas $T_{a r} \mathrm{e}$ $T_{\text {sup }}$, e, portanto, o modelo linear explicou a variância total dos dados dentro de limites aceitáveis para aplicações de estudos urbanos. No entanto, a forte correlação observada corresponde a um período curto de medições (aproximadamente dois meses), e provavelmente esse resultado sofre variações para outros períodos (meses, estações ou anos), visto que estar se tratando com dados relacionados à superfície que podem sofrer ou não variações entre períodos. Desta forma, recomenda-se em trabalhos futuros a extensão dessa análise para períodos mais longos. Por outro lado, na ausência de dados de $T_{a r}$ com uma boa distribuição espacial para estudos de ICU, é possível utilizar os dados de $T_{\text {sup }}$ obtidos por satélite, devido a sua relação espaço-temporal com $T_{a r}$, como mostrado neste estudo. Além disso, o uso combinado de ambos os dados na identificação da ICU torna-se uma perspectiva ainda melhor. Os dados in situ apresentam grande vantagem com relação a sua alta resolução temporal e a possibilidade de se obter uma série histórica. Entretanto, uma grande desvantagem é a distribuição espacial dos dados in situ para realizar um mapeamento adequado, como a exigida por estudos da ICU (Stewart, 2010). Nesse sentido, a dificuldade de realizar estudos de ICU atmosféric poderia ser superada ao utilizar dados de $T_{\text {sup }}$ obtidas de imagens de satélite, como enfatizado por Rajasekar e Weng, (2009).

Um dos impactos ocasionados pelo uso e ocupação de solo se reflete em alterações de suas características físicas. Ao transformar uma área rural em urbana, há uma mudança do $\alpha_{\mathrm{s}}$, o que altera o balanço de radiação em superfície e, consequentemente, $T_{\text {sup }}$. As Figuras $5 \mathrm{a}-5 \mathrm{~b}$ apresentam a distribuição espacial da média mensal de $\alpha_{\mathrm{s}}$ e $T_{\text {sup }}$ para o município de Londrina no mês de agosto de 2011. Observa-se claramente na Figura 5a que os valores de $\alpha_{\mathrm{s}}$ aumentam do centro para a periferia da área delimitada pela mancha urbana. Esse comportamento é esperado, dado que as superfícies urbanas absorvem mais radiação, especialmente nas áreas com maior porcentagem de asfalto e telhado, como no caso dos sítios BIB e BHZ onde os valores de $\alpha_{\mathrm{s}}$ estão entre 0,08 e 0,11 . Os sítios localizados na periferia da mancha urbana (SER e PQE) apresentam valores de $\alpha_{\mathrm{s}}$ um pouco maiores, entre 0,13 e 0,15 , chegando até 0,19 fora da mancha urbana.

Jin et al. (2005) utilizaram dados de $\alpha_{\mathrm{s}}$ do sensor MODIS em três bandas espectrais (visível, infravermelho próximo e todo o espectro de onda curta) para avaliar a região de Pequim (China) e seus arredores. Os $\alpha_{\mathrm{s}}$ obtidos a partir do espectro de onda curta na área urbana de Pequim apresentaram valores de aproximadamente 0,15 , e nas áreas cobertas por floresta e cultivos atingiram valores de cerca de 0,18 . Valores típicos em áreas urbanas sem cobertura de neve variam entre 0,09 e 0,20 (Taha, 1997) e nas áreas rurais entre 0,15 a 0,24 (Krayenhoff e Voogt, 2010; Brest, 1987). Portanto, os valores de $\alpha_{\mathrm{s}}$ encontrados na literatura corroboram os resultados encontrados neste estudo.

Ao avaliar a distribuição espacial de $T_{\text {sup }}$ sobre Londrina no mês de agosto de 2011 (Figura 5b), observa-se um padrão típico de ICU superficial. Para esta parte das análises foi utilizado o software ArcGis 10.1, com o qual foram calculados os valores mínimos e máximos dos pixels contidos no contorno que delimita a mancha urbana, sendo estes valores iguais a $19,5^{\circ} \mathrm{C}$ e $23,3{ }^{\circ} \mathrm{C}$, respectivamente. Os valores máximos estão localizados na porção norte e central da mancha urbana, e os valores mínimos são observados no entorno desta área e no resto do município, com valores até $16,5^{\circ} \mathrm{C}$. Assim, a diferença entre a $T_{\text {sup }}$ máxima e $T_{\text {sup }}$ mínima dentro da mancha urbana denota uma ICU superficial de $3,9^{\circ} \mathrm{C}$.

Targino et al. (2013) identificaram um valor médio de intensidade de ICU atmosférica de $3{ }^{\circ} \mathrm{C}$ na região central de Londrina, em situação de alta-pressão estacionária, com diminuição de intensidade de forma radial em direção às áreas suburbanas, em um padrão semelhante ao observado na Figura 5b. Portanto, a utilização de $T_{\text {sup }}$ constitui uma ferramenta robusta para o monitoramento ou identificação da ICU superficial. Por conseguinte, uma combinação de dados de $T_{\text {sup }}$ obtidos das imagens de satélite com a $T_{a r}$ in situ, permite quantificar e diferenciar este tipo de fenômeno em termos de intensidade, variações sazonais, ciclos diurno e noturno, e também comparar e monitorar ao mesmo tempo inúmeras cidades localizadas em diversas partes do mundo.

A Figura 5c apresenta o IU obtido da combinação linear de $\alpha_{\mathrm{s}} \operatorname{com} T_{\text {sup }}$ através da Equação 4. Maiores valores de IU podem ser usados como referência para identificar áreas densamente urbanizadas. Como pode ser observado, comparando-se qualitativamente a Figura 5c com a Figura 1, esse índice fornece subsídios para identificar corretamente a mancha urbana de Londrina, de uma forma bastante prática e baseada em considerações físicas. Dessa forma, a expansão territorial de uma determinada região urbana poderia ser monitorada de uma forma simples através de uma série espaço-temporal de IU. Adicionalmente, esta série temporal de identificação da expansão urbana poderia ser inserida em modelos climáticos para uma melhor compreensão dos impactos causados pela urbanização local.

O IU inferido neste estudo indica qualitativamente que valores compreendidos entre 18 e 20 são considerados áreas urbanas, entre 16 e 18 áreas suburbanas, e menores a 16, considera-se áreas vegetadas. No entanto, a metodologia para 

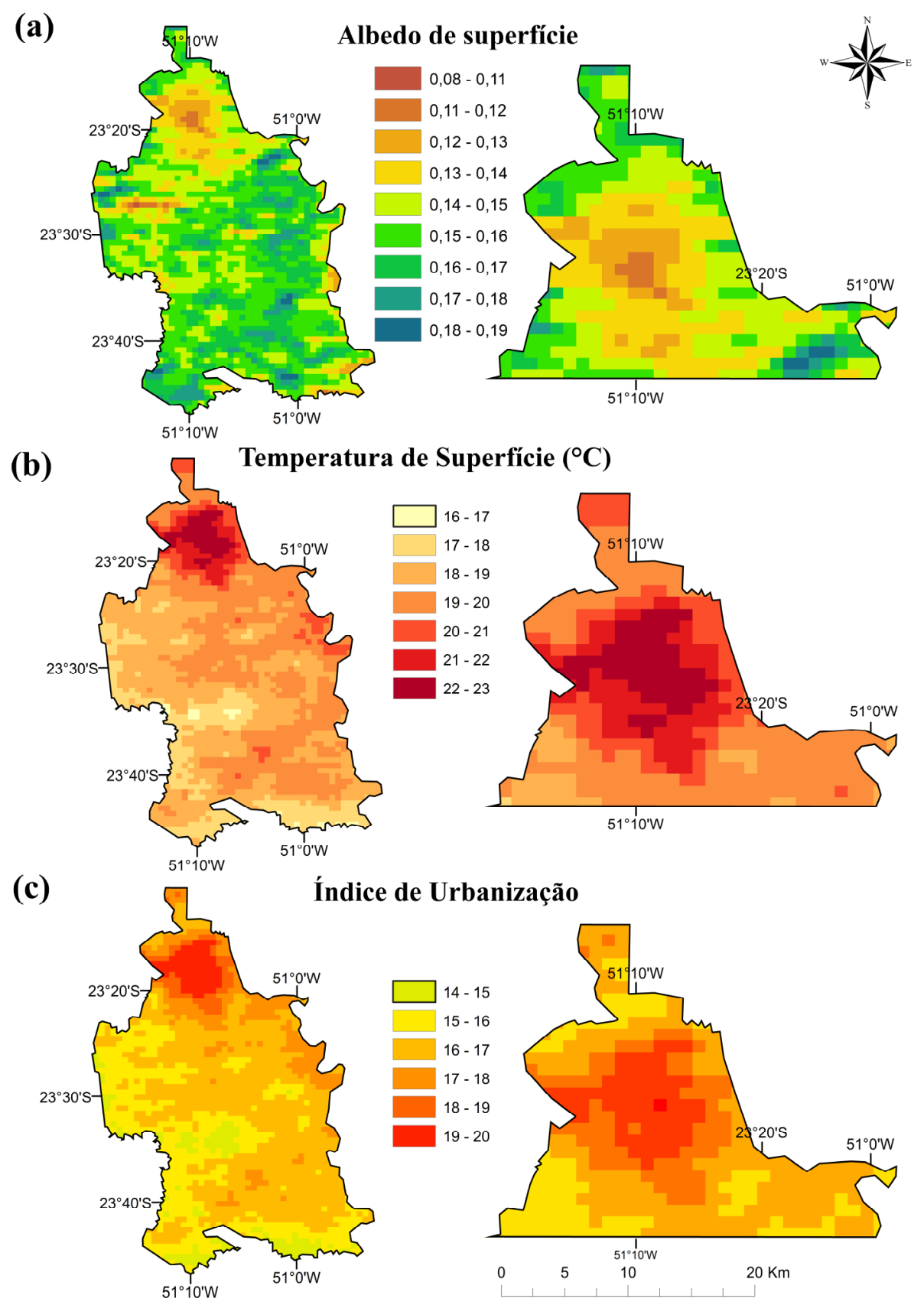

Figura 5 - Distribuição espacial da média mensal de: a) albedo da superfície, b) temperatura de superfície $\left({ }^{\circ} \mathrm{C}\right)$ e c) índice de urbanização $\left({ }^{\circ} \mathrm{C}\right)$ para o município de Londrina no mês de agosto de 2011. Os painéis direitos ilustram ampliações para destacar características sobre as áreas urbanas e sub-urbanas da cidade de Londrina.

extrair IU deve ser aplicada em outros períodos e outras regiões para estabelecer uma generalização desses limiares.

\section{CONCLUSÕES}

Este estudo utilizou dados de sensoriamento remoto provenientes do sensor MODIS para avaliar as variáveis de superfície $\alpha_{\mathrm{s}}$ e $T_{\text {sup }}$ na cidade de Londrina, com o objetivo de fornecer informações suplementares em estudos de ICU. Os dados de $\alpha_{\mathrm{s}}$ e $T_{\text {sup }}$ foram utilizados para obter um índice de urbanização (IU), e foram posteriormente comparados com observações in situ de $T_{a r}$ extraídas de 13 sítios no perímetro urbano de Londrina. De modo geral, as análises dos dados de $T_{\text {sup }}$ apresentaram-se consistentes com as medições in situ de $T_{a r}$ 
durante 72 dias abrangendo os meses de junho, julho, e agosto de 2011. Os coeficientes de correlação entre as variáveis $T_{\text {sup }}$ e $T_{a r}$ foram maiores que 0,70 na maioria dos sítios de monitoramento. Os sítios urbanos apresentaram menores valores de $\alpha_{\mathrm{s}}(0,08-$ $0,12)$ em comparação aos sítios rurais $(0,15-0,19)$. A $T_{\text {sup }}$ é aproximadamente $4^{\circ} \mathrm{C}$ maior nos sítios urbanos comparada com aquelas de sítios suburbanos e rurais.

Os padrões espaciais de $\alpha_{\mathrm{s}}$ e $T_{\text {sup }}$ foram também observados na distribuição espacial do IU. Os valores de IU para áreas mais urbanizadas encontram-se entre 18 e 20, sendo que estes valores vão diminuindo radialmente para áreas mais vegetadas (entre $14-16$ ).

Os dados de sensoriamento remoto utilizados nas análises demonstraram-se bastante úteis na avaliação espaçotemporal da ICU, e também para a avaliação dos impactos de uso do solo decorrentes de mudanças da superfície.

Imagens de satélite são amplamente utilizadas para identificar, classificar e quantificar áreas de uso e ocupação de solo, tais como a localização e mapeamento de áreas urbanas. No entanto, do ponto de vista prático, essa classificação torna-se bastante árdua ou não praticável. Dessa forma, o IU aplicado neste estudo, assim como analisado por Jin et al. (2005) demonstrou-se uma ferramenta eficiente para identificar a mancha urbana de uma cidade de porte médio como Londrina. Além disso, com esse índice, torna-se possível representar de uma forma prática a evolução espaço-temporal das áreas urbanas de cidades com expansão territorial acentuada, propiciando uma avaliação mais detalhada do impacto desse tipo de mudança de cobertura de solo no clima local e regional. No entanto, sugere-se observar os valores do IU em outros períodos e comparar com outras regiões de características similares, de modo a estabelecer um padrão desses valores.

\section{AGRADECIMENTOS}

O primeiro autor agradece pelo suporte financeiro proporcionado pela Organização Meteorológica Mundial (OMM). Os autores também agradecem à Fundação Araucária (convênio número 470/2010) pelo financiamento dos sensores de temperatura do ar utilizados neste estudo, à CAPES pelo custeio das despesas desse artigo via recursos PROAP, à EMBRAPA Soja (Empresa Brasileira de Pesquisa Agropecuária), ao Sistema Meteorológico do Paraná (SIMEPAR), INFRAERO e ao Instituto Agronômico do Paraná (IAPAR) pela cessão de dados meteorológicos. Agradecemos a Guilherme Conor Coraiola (UTFPR - Londrina) pelos dados de cobertura do solo nos sítios utilizados neste estudo, à Universidade Estadual de Londrina e ao IATE Clube de Londrina por participarem na coleta de dados de temperatura do ar, à professora Simone M. S. Costa (CPTEC/INPE) e aos dois anônimos revisores pelos comentários e sugestões ao presente trabalho.

\section{REFERÊNCIAS BIBLIOGRÁFICAS}

ACKER, J.G.; LEPTOUKH, G. Online analysis enhances use of NASA Earth Science Data. Eos, Transactions, American Geophysical Union. v. 88, n. 2, p. 14 - 17, 2007.

AMORIN, M. C. de C. T.; DUBREUIL, V.; QUENOL, H.; NETO, J. L. S.A. Características das ilhas de calor em cidades de porte médio: exemplos de Presidente Prudente (Brasil) e Rennes (França). Confins [Online], $7 \mid 2009$, posto online em 31 Outubro 2009, Consultado o 30 Março 2012. URL :http://confins.revues.org/6070. DOI : 10.4000/confins.6070.

ANDERSON, L. O.; LATORRE, M. L.; SHIMABUKURO, Y. E.; ARAI, E.; JÚNIOR, O. A. de C. Sensor MODIS: uma abordagem geral. INPE-10131-RPQ/752 São José dos Campos - São Paulo, 2003.

ARNFIELD, A. J. Two decades of urban climate research: A review of turbulence, exchanges of energy and water, and the urban heat island. International Journal of Climatology, v. 23, n. 1, p. 1-26, 2003.

ASIMAKOPOULOS, D.N.; DEMOSTHENES, N. Energy and climate in the urban built environment. London: James \& James, 2001. 229 p.

AS-SYAKUR, A. R.; ADNYANA, I. W. S.; ARTHANA, I. W.; NUARSA, I. W. Enhanced Built-Up and Bareness Index (EBBI) for Mapping Built-Up and Bare Land in an Urban Area. Remote Sensing, v. 4, p. 2957-2970, 2012.

BARBIRATO, G. M.; SOUZA, L.C.L.; TORRES, S.C. Clima e cidade: uma abordagem climática como subsídio para estudos urbanos. EDUFAL: Maceió, 164 p., 2007.

BARRY, R. G.; CHORLEY, R. J. Atmosphere, weather and climate. 9 ed. Abingdon: Routledge, 2010. 516 p.

BREST, C.L. Seasonal albedo of an urban/rural landscape from satellite observations, Journal of Applied Meteorology, v. 26, p.1169-1187, 1987.

CÂMARA, G.; SOUZA, R.C.M.; FREITAS, U. M.; GARRIDO, J. SPRING: Integrating remote sensing and GIS by object - oriented data modeling. Computers \& Graphics, v. 20, n.3, p. $395-403,1996$.

CERMAK, J. E.; DAVENPORT, A. G.; PLATE, E. J.; VIEGAS, E. G. Wind climate in cities. Kluwer Academic Publichers: Dordrecht, 1995. p. 81.

CHEN, X.L.; ZHAO, H. M.; LI, P. X.; YIN, Z. Y. Remote sensing image-based analysis of the relationship between urban heat island and land use/cover changes. Remote Sensing of Environment, v. 104, p. 133 - 146, 2006.

CIHLAR, J. Land cover mapping of large areas from satellites: status and research priorities. International Journal of Remote Sensing, v. 21, n. 6 e 7, p. 1093- 1114, 2000.

COLL, C.; CASELLES, V.; GALVE, J. M.; VALOR, E.; NICLÒS, R.; SÁNCHEZ, J. M.; RIVAS, R. Ground 
measurements for the validation of land surface temperatures derived from AATSR and MODIS data. Remote Sensing of Environment, v. 97, p. 288 - 300, 2005.

DOUSSET, B.; GOURMELON, F. Satellite multi-sensor data analysis of urban surface temperatures and landcover. ISPRS Journal of Photogrammetry and Remote Sensing, v. 58, p. $43-54,2003$.

DOUSSET, B.; GOURMELON, F.; LAAIDI, K.; ZEGHNOUN, A.; GiRAUDET, E.; BRETIN, P.; MAURI, E.; VANDENTORREN, S. Satellite monitoring of summer heat waves in the Paris metropolitan area. International Journal of Climatology, v. 31, p.313-232, 2011.

EOSDIS. Earth Observing System Data and Information System. Earth Observing System Clearing HOuse (ECHO) /Reverb, Version 10 [online application]. Greenbelt, MD: EOSDIS, Goddard Space Flight Center (GSFC) National Aeronautics and Space Administration (NASA). 2009. Disponível em: $<$ http://reverb.earthdata.nasa.gov $>$. Acesso em: 11 mar. 2013.

FERREIRA, M.J. Estudo do balanço de energia na superfície da cidade de São Paulo. 2010. 183 f. Tese (Doutorado em Meteorologia) - Universidade de São Paulo - Instituto de Astronomia, Geofísica e Ciências Atmosféricas, 2010.

FERREIRA, M. J.; OLIVEIRA, A. P.; SOARES, J. Anthropogenic heat in the city of São Paulo, Brazil. Theoretical and Applied Climatology, 2011. Doi:10.1007/ s00704-010-0322-7.

FREY, C.M.; PARLOW, E.; VOGT, R.; HARHASH, M.; MOHAMMAD, M.; WAHAB, A. Flux measurements in Cairo. Part 1: in situ measurements and their applicability for comparison with satellite data. International Journal of Climatology, v. 31, p. 218-231, 2011.

FRIEDL, M. A.; MENASHE, D.S.; TAN, BIN.; SCHNEIDER, A.; RAMANKUTTY, N.; SIBLEY, A.; HUANG, X. MODIS Collection 5 global land cover: Algorithm refinements and characterization of new datasets. Remote Sensing of Environment., v. 114, p. 168 - 182, 2010.

FUJIBE, F. Urban warming in Japanese cities and its relations to climate change monitoring. International Journal of Climatology, v. 31, p.162-173, 2011.

GALLO, K. P.; McNAB, A. L.; KARL, T. R.; BROWN, J. F.; HOOD, J. J.; TARPLEY, J.D. The use of NOAA AVHRR data for assessment of the urban heat island effect. Journal of Applied Meteorology, v. 32, p.899-908, 1993.

GARTLAND, L. Heat islands: understanding and mitigating heat in urban areas. London: Earthscan, p. 27 -35, 2008. 215p.

GIRIDHARAN, R.; GANESAN, S.; LAU, S. S. Y. Daytime urban heat island effect in high-rise and high-density residential developments in Hong Kong. Energy and Buildings, v.36, p. 525-534, 2007.

HU, Y.; JIA, G. Influence of land use change on urban heat island derived from multi-sensor data. International Journal of Climatology, v. 30, n. 9, p. 1382-1395, 2010.

IMHOFF, M. L.; ZHANG, P.; WOLFE, R. E.; BOUNOUA, L. Remote sensing of the urban heat island effect across biomes in the continental USA. Remote Sensing of Environment, n. 114, p. $504-513.2010$.

INSTITUTO BRASILEIRO DE GEOGRAFIAE ESTATÍSTICA (IBGE). Estimativas populacionais para os municípios brasileiros em 01.07.2012, 2012. Disponível em: <http:// www.ibge.gov.br/home/estatistica/populacao/estimativa2012/ estimativa dou.shtm>. Acesso em: 20 abr. 2013.

JIN, M.; DICKINSON, R.E.; ZHANG, D. L. The Footprint of Urban Areas on Global Climate as Characterized by MODIS. Journal of Climate, v.18, p. 1551 - 1565, 2005.

JIN, M.S. Developing an index to measure urban heat island effect using satellite land skin temperature and land cover observations. Journal of Climate, v. 25, n. 18, p. $6193-$ 6201, 2012.

KAWAMURA, M.; JAYAMANNA, S.; TSUJIKO, Y.; SUGIYAMA, A. Comparison of urbanization of four Asian cities using satellite data. Journal of Environmental System \& Engineering, n. 608, v. 7 - 9, 1998.

KRAYENHOFF, E.S.; VOOGT, J.A. Impacts of urban albedo increase on local air temperature at daily-annual time scales: model results and synthesis of previous work. Journal of Applied Meteorology and Climatology, v. 49, n.8, p. 1634 - 1648, ago. 2010.

KRÜGER, E. L.; MINELLA, F. O.; RASIA, F. Impact of urban geometry on outdoor thermal comfort and air quality from field measurements in Curitiba, Brazil. Build Environment, v. 46, p. 621, 2011.

LAI, L.W.; CHENG, W.L. Air quality influenced by urban heat island coupled with synoptic weather patterns. Science of The Total Environment, v. 407, p. 2724 - 2733, 2009.

LEWIS, P.; BARNSLEY, M. J. Influence of the sky radiance distribution on various formulations of the earth surface albedo, In: Proc. Conf. Phys. Meas. Sign. Remote Sens., 1994, Val d'Isere, France. p. 707 - 715.

LOMBARDO, M. A. Ilha de calor nas metrópoles: o exemplo de São Paulo - São Paulo: HUCITEC, 1985.

LUCENA A. J.; ROTUNNO FILHO, O. C.; FRANÇA, J. R. A.; PERES, L. F.; XAVIER, L. N. R. Urban climate and clues of heat island events in the metropolitan area of Rio de Janeiro. Theoretical and Applied Climatology, v. 111, p. 497-511, 2013.

McKINNEY, M. L. Urbanization, biodiversity and conservation. Bioscience. v. 52, n. 10, p. $883-890,2002$. 
McROBERTS, R. E.; WENDT, D.G.; NELSON, M.D.; HANSEN, M.H. Using a land cover classification based on satellite imagery to improve the precision of forest inventory area estimates. Remote Sensing of Environment, v. 81, p. $36-44,2002$.

NICHOL, J. E. Visualisation of urban surface temperatures derived from satellite images. International Journal of Remote Sensing, v. 19, n. 9, p. 1639 - 1649, 1998.

OKE, T. R. City size and the urban heat island. Atmospheric Environment, v. 7, p. $769-779,1973$.

OKE, T.R. The energetic basis of the urban heat island. Quarterly Journal of the Royal Meteorological Society, v. 108, p. 1-24, 1982.

OMETTO, J. C. Bioclimatologia vegetal. São Paulo: Ceres. $1981.425 \mathrm{p}$.

PARTECKE, J.; SCHWABL, I.; GWINNER, E. Stress and the city: urbanization and its effects on the stress physiology in European blackbirds. Ecological Society of America: Ecology, v. 87, n. 8, p. 1945-1952, 2006.

PENG, S.; PIAO, S.; CIAIS, P.; FRIEDLINGSTEIN, P.; OTTLE, C.; BRÉON, F.M.; -, H.; ZHOU, L.; MYNENI, R.B. Surface urban heat island across 419 global big cities. Environmental Science \& Technology, v. 46, p. 696 - 703, 2012.

RAJASEKAR, U.; WENG, Q. Urban heat island monitoring and analysis using a non-parametric model: A case study of Indianapolis. ISPRS Journal of Photogrammetry and Remote Sensing, v. 64, p. 86-96, 2009.

SCHAAF, C. Tools for MODIS BRDF/Albedo Products: General tools for MODIS L3 data. Actual (Blue-Sky) albedo computation. Last 5 July 2006. Disponível em: $<$ http://www-modis.bu.edu/brdf/userguide/tools.html $>$. Acesso em 03 abr. 2011.

STEWART, I. D. A systematic review and scientific critique of methodology in modern urban heat island literature. International Journal of Climatology, 2010.

STRAHLER, A.H.; LUCHT, W.; SCHAAF, T. T.; GAO, F.; LI, X.; MULLER, J. P.; LEWIS, P.; BARNSLEY, M. J. MODIS BRDF/ Albedo Product: Algoritm theoretical basis document Version 5.0., 1999. p. 9-10.

TAHA, H. Urban climates and heat islands: albedo, evapotranspiration, and anthropogenic heat. Energy and Buildings, v. 25, p. 99 - 103, 1997.

TARGINO, A. C.; KRECL, P.; CORAIOLA, G. C. Effects of the large-scale atmospheric circulation on the onset and strength of urban heat islands: a case study. Theoretical and Applied Climatology, doi:10.1007/s00704-013-0989-7, 2013.
UNGER, J. Intra-urban relationship between surface geometry and urban heat island: review and new approach. Climate Research, v. 27, p. 253-264, 2004.

UN-PD. POPULATION DIVISION OF THE DEPARTMENT OF ECONOMIC AND SOCIAL AFFAIRS OF THE UNITED NATIONS SECRETARIAT. World population prospects: the 2007 revision population database, 2007. Disponível em: <http://esa.un.org/unup>. Acesso em: 31 mar. 2012.

UPMANIS, H.; ELIASSON, I.; LINDQVIST, S. The influence of green areas on nocturnal temperatures in a high latitude city (Göteborg, Sweden). International Journal of Climatology, v. 18, p. 681-700, 1998.

VOOGT, J.A.; OKE, T.R. Thermal remote sensing of urban climates. Remote Sensing of Environment, v. 86, p. 370-384, 2003.

WAN, Z.; DOZIER, J. A generalized split - window algorithm for retrieving land - surface temperature from space. . IEEE Transactions On Geoscience And Remote Sensing, v. 34, n.4, p. 892 - 904, July 1996.

WAN, Z.; ZHANG, Y.; ZHANG, Q. Quality assessment and validation of the MODIS global land surface temperature. International Journal of Remote Sensing, v. 25, n. 1, p. $261-274,2004$.

WENG, Q.; LU, D.; SCHUBRING, J. Estimation of land surface temperature - vegetation abundance relationship for urban heat island studies. Remote Sensing of Environment, v. 89, n. 4, p. $467-483,2004$.

WILDI, W.; DOMINIK, J.; LOIZEAU, J.L.; THOMAS, R. L.; FAVARGER, P.Y.; HALLER, L.; PERROUD A.; PEYTREMANN C. River, reservoir and lake sediment contamination by heavy metals downstream from urban areas of Switzerland. Lake And Reservoir Management, v. 9 , p. $75-87,2004$.

$\mathrm{XU}, \mathrm{H}$. A new index for delineating built-up land features in satellite imagery. International Journal of Remote Sensing, v. 29, p. 4269-4276, 2008.

YANG, F.; LAU, S. S. Y.; QIAN, F. Summertime heat island intensities in three high-rise housing quarters in inner-city Shanghai China: Building layout, density and greenery. Build Environment, Hong Kong, v. 45, p. 115-134, 2010. ZHA, Y.; GAO, J.; NI, S. Use of normalized difference builtup index in automatically mapping urban areas from TM imagery. International Journal of Remote Sensing, v. 24, p. 583 - 594, 2003. 